\title{
From BASIS to MIRACLES: Benchmarking and perspectives for high-resolution neutron spectroscopy at the ESS
}

\author{
Nikolaos Tsapatsaris ${ }^{1,2, a}$, Peter K. Willendrup ${ }^{3,4}$, Ruep E. Lechner ${ }^{2}$ and Heloisa N. Bordallo ${ }^{1,2, a}$ \\ ${ }^{1}$ Niels Bohr Institute, The University of Copenhagen, Copenhagen 2100, Denmark \\ ${ }^{2}$ European Spallation Source, Lund 22100, Sweden \\ ${ }^{3}$ Technical University of Denmark, 2800 Kongens Lyngby, Denmark \\ ${ }^{4}$ European Spallation Source, Data Management and Software Centre, Copenhagen 2100, Denmark
}

\begin{abstract}
Results based on virtual instrument models for the first high-flux, high-resolution, spallation based, backscattering spectrometer, BASIS are presented in this paper. These were verified using the Monte Carlo instrument simulation packages McStas and VITESS. Excellent agreement of the neutron count rate at the sample position between the virtual instrument simulation and experiments was found, in both time and energy distributions. This achievement was only possible after a new component for a bent single crystal analyser in McStas, using a Gaussian approximation, was developed. These findings are pivotal to the conceptual design of the next generation backscattering spectrometer, MIRACLES at the European Spallation Source.
\end{abstract}

\section{Introduction}

The planned construction of the European Spallation Source (ESS) marks a new era for the international neutron scattering community [1].

The time of flight backscattering spectrometer MIRACLES (Multiple Resolution Analyser Crystal for Life and Energy Science) to be proposed in 2015 has the unique possibility of utilizing the full length of the ESS pulse. By using $\operatorname{Si}(111)$ analyser banks and a chopper cascade, the instrument will offer tuneable elastic energy resolution in the range of $3-32 \mu \mathrm{eV}$ and a flexible dynamic range larger than $1000 \mu \mathrm{eV}$. Furthermore, by using different analyser crystals, for instance $\mathrm{Si}(311)$, the $\mathrm{Q}$ range can be extended to $3.8 \AA^{-1}$. This will allow the determination of dynamical phenomena taking place at a length scale of $1.5 \AA$. Consequently, the resolution map and energy range covered by high-energy resolution direct geometry time of flight (TOF), triple axis (TAS) spectrometers, spin-echo and reactor backscattering spectroscopy can be bridged. It is envisaged that the high source brilliance, along with a pulse length of $2.86 \mathrm{~ms}$ and the slow neutron pulse repetition rate of $14 \mathrm{~Hz}$, will permit the development of an instrument with unprecedented flux [2].

MIRACLES will bring new insights into scientific topics related to diffusion [3], molecular rotation [4], critical scattering and tunneling in soft and hard condensed matter and biologically relevant materials [5], as well as low energy excitations in magnetic systems [6]. In addition, the time domain of $1 \mathrm{ps}-1 \mathrm{~ns}$ that can be covered with reasonable computational effort by Molecular Dynamics (MD) modeling is ideally suited to that offered by MIRACLES. Moreover, the combined

${ }^{a}$ Corresponding authors: n.tsapatsaris@nbi.ku.dk; bord allo@nbi.ku.dk use of Infrared spectroscopy (IR), Nuclear Magnetic Resonance (NMR), Raman scattering and dielectric spectroscopy with results obtained from MIRACLES, will allow the characterization of a plethora of phenomena seen in modern materials. Being part of the unique scientific ecosystem of the ESS, MIRACLES will help open up new scientific horizons. For example, water dynamics that can be probed by quasi-elastic neutron scattering (QENS) on the $1 \mathrm{ps}-1 \mathrm{~ns}$ timescale play an important role in drug release characteristics and molecular conformation, of novel injectable drug delivery media made from thermoresponsive polymer micro-capsules $[7,8]$ or widely used analgesics [9-11]. Moreover, using MIRACLES it will be possible to effectively probe the multiple energy states of water held by? surfaces of a clay mineral. This knowledge provides important information for the design of safer hydraulic barriers where water and contaminant transfer must be controlled, for instance, for the containment of industrial, municipal, and hazardous wastes [12]. Precise knowledge of the microscopic structure of bulk water and water under confinement is of utmost importance for most chemists and molecular biologists [13].

For the purpose of serving as a platform for the development of MIRACLES at the ESS, the BASIS instrument [14], located at the Spallation Neutron Source (SNS) at Oak Ridge, USA, was benchmarked with Monte Carlo simulations. The near-backscattering spectrometer BASIS, has proven to be extremely successful in research topics that span the disciplines of biology, chemistry and soft and hard condensed matter $[14,15]$. The clear advantage of this instrument is that it allows for the elucidation of overlapping complex dynamical phenomena occurring at markedly different timescales. It is the first high-flux, high-resolution, indirect geometry neutron spectrometer to provide a dynamic range of $\pm 250 \mu \mathrm{eV}$

This is an Open Access article distributed under the terms of the Creative Commons Attribution License 4.0, which permits unrestricted use, distribution, and reproduction in any medium, provided the original work is properly cited. 
with an energy resolution of $\Delta E=3 \mu \mathrm{eV}$ or $\Delta E / E=$ $1.5 \times 10^{-3}$.

The two key features of the BASIS instrument operation are: making use of the temporal structure of the SNS pulsed source and the incorporation of physically bent single crystals, which reflect near the Bragg limit: $\theta \approx 86-89^{\circ}$. Consequently, for the purpose of simulating the BASIS instrument and in order to validate the design of MIRACLES, a new spherically bent single crystal component was developed in McStas [16]. This new feature also allowed comparisons of simulation results obtained with VITESS [17]. In summary, we present successful instrument simulations of the BASIS instrument, which show good agreement with published results [14] and finally, we give a glimpse of the future performance of MIRACLES at the ESS, in terms of flux and energy resolution.

\section{Virtual instrument setup}

A virtual instrument was developed in McStas using the published instrumental parameters of BASIS as input [14]. McStas 2.0, was used for the simulations and executed on the Data Management and Software Centre (DMSC) at the Niels Bohr Institute in the University of Copenhagen. This allowed the collection of sufficient simulation statistics, using $10^{12}$ neutron trajectories over a wavelength range of $\Delta \lambda=0.5-30 \AA$, within 24 hours. In addition, an identical virtual instrument setup was developed in VITESS 3.1. In this case the simulations were run on a personal computer (MacOS10.6) and reasonable statistics, using $10^{10}$ neutron trajectories, were obtained for a wavelength band of $\Delta \lambda=$ $6-7 \AA$ in 10 hours.

\section{Primary spectrometer}

The instrument layout is shown in Fig. 1. The distance from the moderator to the sample position is $84 \mathrm{~m}$. The moderator illuminating the BASIS guide at SNS is a decoupled supercritical hydrogen centre-linepoisoned moderator. At SNS, the moderator emission time of $6.271 \AA$ neutrons, after protons hit the target, is approximately $60 \mu \mathrm{s}$ for a pulse repetition frequency and moderator beam size of $60 \mathrm{~Hz}$ and $\mathrm{H}=10 \mathrm{~cm} / \mathrm{W}=12 \mathrm{~cm}$ respectively. The original SNS_moderator description file, available in McStas, makes use of linear interpolation into statistically limited, spectral histograms, produced using the MCNPX code, which resulted in artefacts in the long-wavelength region. To remedy this issue, the "ESS_ moderator_ short" description file, with the same moderator parametrisation as the SNS, was used instead. This is possible because both moderators operate with a similar physical principle and the underlying analytics follow the same master curve.

The wavelength band and frame overlap disk choppers were rotated with the same frequency as the SNS source. The dimensions of the chopper windows are described in the BASIS publication [14]. A phase offset was applied to the components describing the chopper geometry in order to permit a neutron wavelength band centred at $6.267 \AA$. This value corresponds to the Bragg reflection of $\mathrm{Si}(111)$ crystal with a $2 \theta=88^{\circ}$.

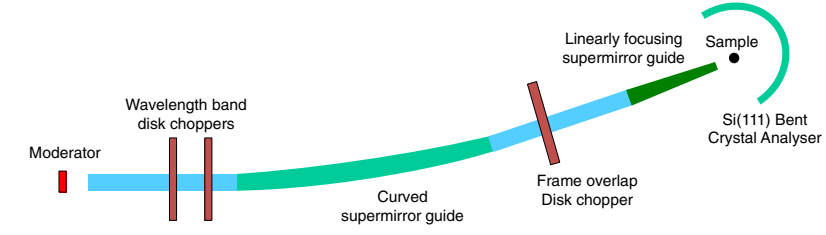

Figure 1. The schematic layout of the BASIS spectrometer. The guide entrance is illuminated by a decoupled supercritical hydrogen poisoned moderator and the sample position at $84 \mathrm{~m}$ is at the end of a linearly tapered guide.

Table 1. Super-mirror reflectivity data used in the simulations.

\begin{tabular}{|c|c|c|}
\hline $\begin{array}{c}\mathrm{m}= \\
\mathrm{Qmax} / \mathrm{Qmax}(\mathrm{Ni})\end{array}$ & $\begin{array}{c}\text { Reflectivity at } \\
\mathrm{Q}=\mathrm{m}^{*} \mathrm{Qc}(\mathrm{Ni})\end{array}$ & $\begin{array}{c}\text { Width of cut-off } \\
\text { region }(\alpha)[\AA]\end{array}$ \\
\hline 1 & 0.99 & 0.003 \\
\hline 1.5 & 0.97 & 0.003 \\
\hline 2 & 0.95 & 0.003 \\
\hline 2.5 & 0.92 & 0.003 \\
\hline 3.6 & 0.78 & 0.003 \\
\hline
\end{tabular}

The guide geometry was reproduced as described in the instrument publication [14]. In addition, to allow the incorporation of the focusing sections in the simulation packages, their tapering angles were calculated separately.

The slope for the horizontal planes was calculated using a length of $650 \mathrm{~cm}$, entrance $10 \mathrm{~cm}$ and exit $3.25 \mathrm{~cm}$. This results to a slope angle of $0.297495^{\circ}$.

Similarly, for the vertical focusing plane for a length of $800 \mathrm{~cm}$, entrance of $12 \mathrm{~cm}$ and exit $3.25 \mathrm{~cm}$ the slope is $0.313333^{\circ}$.

For the simulation of the guide mirror surfaces, Mirrotron reflectivity data [18] was used as a template for creating reflectivity files in McStas and VITESS using the following relations resulting from a phenomenological fit to experimental data:

$\mathrm{R}=1 / 2 \mathrm{R}_{0}\left\{1-\tanh \left[\left(\mathrm{Q}-\mathrm{m} \mathrm{Q}_{\mathrm{c}, \mathrm{Ni}}\right) / \mathrm{W}\right]\right\}\left[1-\alpha\left(\mathrm{Q}-\mathrm{Q}_{\mathrm{c}}\right)\right]$

with $\alpha=\left(\mathrm{R}_{\mathrm{m}}-\mathrm{R}_{0}\right) /\left(\mathrm{m} \mathrm{Q} \mathrm{c}, \mathrm{Ni}-\mathrm{Q}_{\mathrm{c}}\right)$.

Where, $\mathrm{Q}$ is the magnitude of the scattering vector ( $\hbar \mathrm{Q}$ is the momentum transfer), $\mathrm{Qc}(\mathrm{Ni})$ is the $\mathrm{Q}$ value corresponding to the critical angle of Nickel, $\mathrm{R}$ denotes the guide reflectivity, $R_{m}$ the reflectivity at the critical angle according to the $m$ coating of the guide and $R_{0}$ the reflectivity at low angles. The calculated reflectivity values are shown in Table 1.

\section{Secondary spectrometer}

A schematic view of the secondary spectrometer geometry can be seen in Fig. 2. In order to describe the energy response of the crystal analysers, a new component was developed (Sect. 3.2). We reduced the computational load incurred by the simulations by restricting the analyser coverage to $\pm 10^{\circ}$ in the horizontal and vertical scattering planes. The collimation optics, aluminum scattering contributions from vacuum chamber and sample environment were not simulated. 


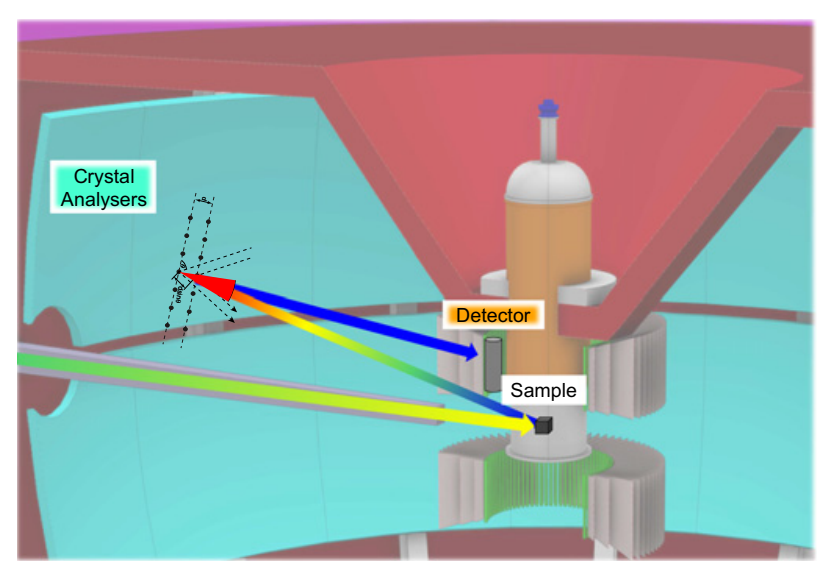

Figure 2. The secondary spectrometer layout, which is typical of spallation-source based TOF - backscattering instruments such as BASIS.

A more detailed description of these background contributions will be later included in a study of the completed MIRACLES instrument.

As a sample reference, a can of annular geometry with a $2.9 \mathrm{~cm}$ diameter, a thickness of $1 \mathrm{~mm}$ and absorption and scattering cross-sections, corresponding to pure vanadium, was used.

\section{Results and discussion}

\subsection{Neutron beam at sample position}

The flux of the BASIS virtual instrument was first simulated at the sample position located at a distance of $84 \mathrm{~m}$ from the moderator surface.

As in the real instrument, a wavelength band of $\Delta \lambda=$ $0.5 \AA$, which was centred at the wavelength of $\lambda=6.4 \AA$, was defined by the chopper cascade. A beam with a crosssection of $3.1 \times 3.1 \mathrm{~cm}^{2}$ was then extracted. The spatial neutron distribution, as well as the integrated neutron intensity, was subsequently collected for $1 \mathrm{sec}$. The results of Fig. 3 (left) indicate that with McStas the simulated flux at the sample position was $1.33 \times 10^{8} \mathrm{n} / \mathrm{s}$. This corresponded to a value, which was within $5 \%$ compared to the published flux of $1.30 \times 10^{7} \mathrm{n} /\left(\mathrm{cm}^{2} \mathrm{~s}\right)$ [14], when the accelerator power was $500 \mathrm{~kW}$.

Similarly, the instrument was simulated using VITESS Fig. 3 (right). An integrated flux of $1.31 \times 10^{8} \mathrm{n} / \mathrm{s}$ or $1.36 \times 10^{7} \mathrm{n} /\left(\mathrm{cm}^{2} \mathrm{~s}\right)$ was simulated over the measured area. The integrated flux and 2-D neutron flux distribution, of both the McStas and Vitess simulation results compare favourably to the published value of $1.3 \times 10^{7} \mathrm{n} /\left(\mathrm{cm}^{2} \mathrm{~s}\right)$ and images therein [14].

\subsection{Energy response of a spherically bent crystal analyser}

While the use of dynamical scattering theory [19] provides a quantitative way to predict the energy response of deformed crystals, it is generally computationally very expensive in a Monte Carlo setting. However, the energy response of a bent single crystal can also be phenomenologically approximated with a Gaussian function. With this

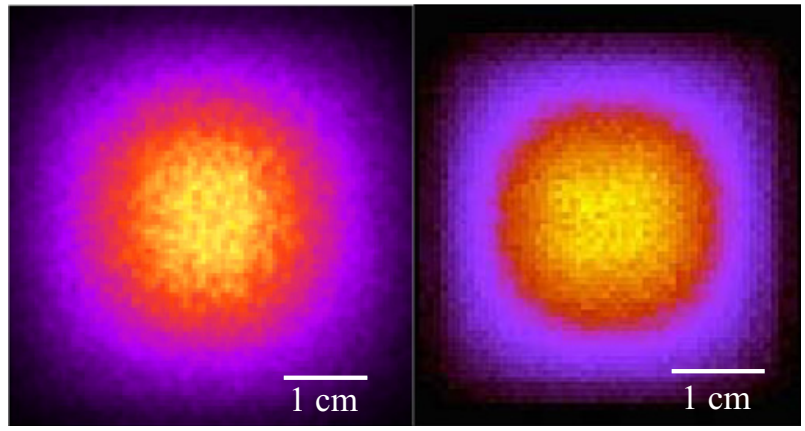

Figure 3. Beam profile at the sample position of the BASIS virtual instrument in McStas (left) and VITESS (right) for a wavelength band of $\Delta \lambda=0.5 \AA$ centred at $\lambda=6.4 \AA$. The area covered by the beam is approximately $9.6 \mathrm{~cm}^{2}$. The colour scale was selected to allow easy comparison with results in the BASIS publication [14].

approach, the distribution of neutron scattering probability versus the outgoing neutron scattering energy vector can be obtained for every neutron trajectory. The FWHM of the Gaussian distribution attempts to describe, in a computationally inexpensive fashion, the cumulative contributions of the uncertainty of the d-spacing due to lattice strains, primary and secondary extinction, incident beam divergence and deviations from the Bragg reflection angle. In our treatment of the analyser dynamic energy response, only the uncertainty of the crystal lattice spacing and the incident beam angular spread was considered. The remaining effects are intrinsically included in the shape of the energy response of the crystal analyser.

We now consider a basic treatment of the relative wavelength uncertainty $\frac{d \lambda_{a}}{\lambda}$ of a crystal analyser using Bragg's law. First, the effect of incident angle uncertainty, or the "apparent" mosaicity, to the wavelength resolution is calculated.

$$
\begin{gathered}
n \lambda=2 d \sin \theta, \quad \text { with } n=1 \\
\frac{\partial \lambda_{M O S A I C}}{\partial \theta}=2 d \cos \theta \\
\partial \lambda_{M O S A I C}=2 d \cos \theta \partial \theta
\end{gathered}
$$

$$
\frac{\partial \lambda_{M O S A I C}}{\lambda}=\frac{2 d \cos \theta \partial \theta}{2 d \sin \theta} \rightarrow \frac{\partial \lambda_{M O S A I C}}{\lambda}=\cot \theta \partial \theta .
$$

Similarly, we now consider the effect of lattice uncertainty to the wavelength resolution.

$$
\begin{gathered}
\frac{\partial \lambda_{\text {lattice }}}{\partial d}=2 \sin \theta \\
\partial \lambda_{\text {lattice }}=2 \sin \theta \partial d \\
\frac{\partial \lambda_{\text {lattice }}}{\lambda}=\frac{2 \sin \theta \partial \mathrm{d}}{2 \mathrm{~d} \sin \theta} \rightarrow \frac{\partial \lambda_{\text {lattice }}}{\lambda}=\frac{\partial \mathrm{d}}{\mathrm{d}} .
\end{gathered}
$$

Assuming statistical independence of the angular spread uncertainty $\partial(\theta)$ and the spread of the crystal analyser 
lattice constant $\mathrm{d},\left[\frac{\partial(d)}{d}\right]$, the cumulative effect of the contributions from Eqs. (6) and (9) can be expressed as:

$$
\left(\frac{\partial \lambda_{a}}{\lambda}\right)^{2}=\left[\frac{\partial(d)}{d}\right]^{2}+[\cot \theta \partial(\theta)]^{2} .
$$

Solving for $\partial \lambda_{a}$ and using delta $(\delta)$ instead of the differential notation $(\partial)$ eventually results in:

$$
\delta \lambda_{a}^{2}=[2 \sin \theta \delta d]^{2}+4 d^{2}\left(1-\sin \theta^{2}\right) \delta \theta^{2} .
$$

Having calculated the two main contributions to the wavelength uncertainty of the crystal analyser, and thus the main changes of the neutron energy distribution upon scattering, the following steps describe the most important functions of the analyser component in McStas. It shall be noted that the parameter values and various constants used in the component module were taken from the BASIS instrument publication [14] and used throughout.

\section{Parameter constants}

Lattice spacing uncertainty: $\frac{\delta(d)}{d}=d_{-}$spread $=3.5 \times$ $10^{-4}$ [14], elastic scattering vector $Q=2.00389 \AA^{-1}$, analyser $\mathrm{d}$-spacing $=d_{\text {s }}$ space $=3.1355 \AA \mathrm{Si}(111)$ for $2 \theta=88^{\circ}$, crystal analyser radius $=2.5 \mathrm{~m}$, crystal reflectivity $\mathrm{R}_{0}=1$, Mosaic spread $=21$ arcmin.

The following pseudo code describes the operation of the component.

1. Convert analyser d-spacing spread and mosaic spread from FWHM to standard deviation $\left(\sigma \_\delta d\right.$ and $\left.\sigma \_m o s\right)$ to facilitate direct incorporation to the Gaussian function, using

$$
\sigma=\frac{F W H M}{\sqrt{8 \ln 2}} .
$$

2. Project the incident neutron vector on the normal plane of reflection.

3. Calculate the neutron wavelength difference $\mathrm{d} \lambda_{\mathbf{n}}=$ $\lambda_{\mathbf{B}}-\lambda_{\mathbf{n}}$ between Bragg condition wavelength and incident neutron wavelength.

4. Determine the analyser wavelength width $\delta \lambda_{a}$ or simply 1-sigma $\left(\sigma_{\lambda \mathbf{a}}\right)^{2}$ from Eq. (11).

$$
\begin{aligned}
\left(\sigma_{\lambda \mathbf{a}}\right)^{2}= & 4(\sin \theta)^{2} \sigma_{-} \delta(d)^{2}+4 d_{-} \text {space }^{2} \\
& \times\left[1.0-(\sin \theta)^{2}\right] \sigma_{-} \text {mos }^{2} .
\end{aligned}
$$

5. Use a probability of reflection as a function of energy following a Gaussian distribution.

$$
\text { p_reflect }=\mathrm{R}_{0}^{*} \exp \left\{-\left(\mathrm{d} \lambda_{\mathbf{n}}\right)^{2} /\left[2\left(\sigma_{\lambda_{\mathbf{a}}}\right)^{2}\right]\right\} .
$$

6. Propagate the probability of reflection.

$$
p \_n e w=p \_r e f l e c t \times p \_o l d .
$$

7. Repeat the treatment for the next neutron trajectory.

This component will be made available in the next version release of McStas.

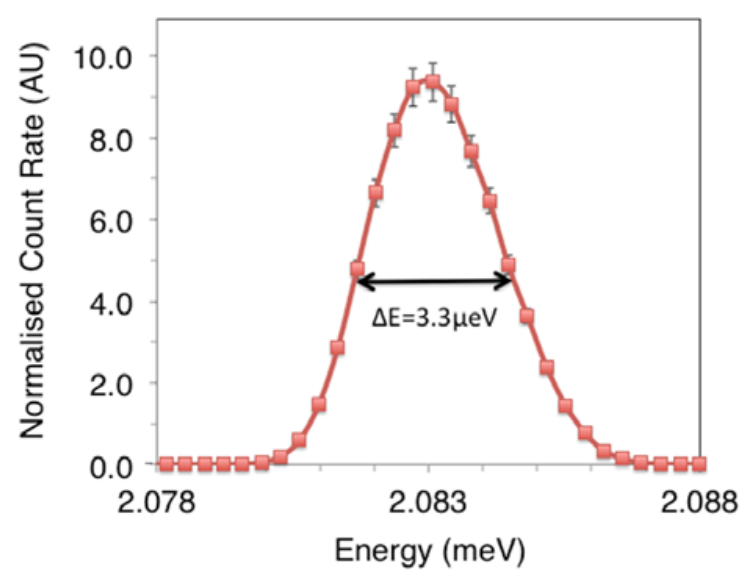

Figure 4. Dynamic energy response of the analyser recorded by the detectors of the virtual BASIS instrument in McStas.

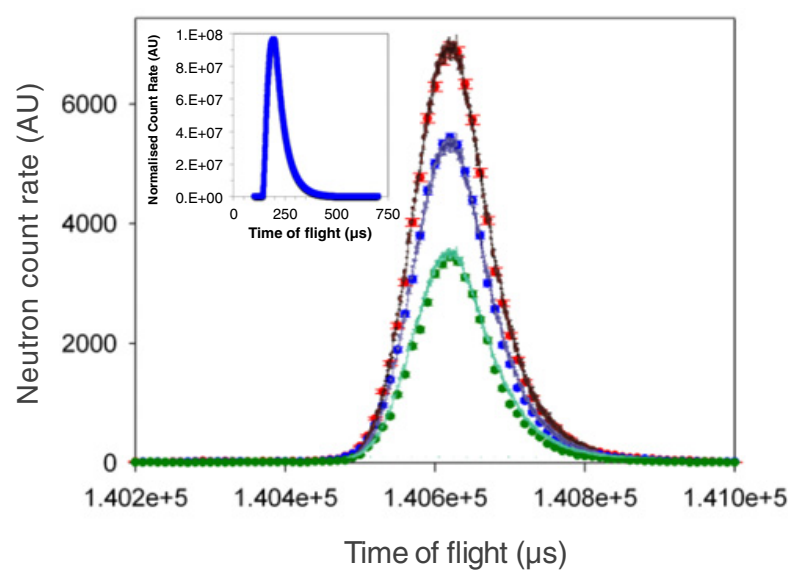

Figure 5. Time of flight data from simulation and experiment. Symbols correspond to data points collected by the detectors of the BASIS instrument for different vanadium sample transmissions (green open circles $98 \%$, blue crosses $96 \%$, red circles 94\% [14]). Solid lines depict TOF data extracted from the virtual BASIS instrument using McStas simulations. The amplitude of the solid lines has been scaled for the different sample transmissions. Inset: Simulation of the time of flight neutron distribution at the moderator surface.

\subsection{Virtual instrument performance}

The neutronic performance of the virtual instrument code using the new component was compared to experimental data in energy and time of flight as shown in Figs. 4 and 5 . The energy response of the analyser produces a FWHM of $3.3 \mu \mathrm{eV}$ versus $3-3.5 \mu \mathrm{eV}$ as reported in reference [14] and shown in Fig. 4. The slight asymmetry that can be seen in the elastic response is caused by two overlapping effects. First, the offset angle of $88^{\circ}$ of the analysers creates a small difference in the time-of-flight path between neutrons travelling from the top and bottom part of the analyser. An additional asymmetric contribution, which is clearly visible in Fig. 5, is caused by the long tail of the SNS spallation source (Fig. 5, inset). As shown, these effects are faithfully reproduced by our simulations.

Figure 5 depicts the instrument performance in arbitrary units as a function of neutron time of flight arrival 


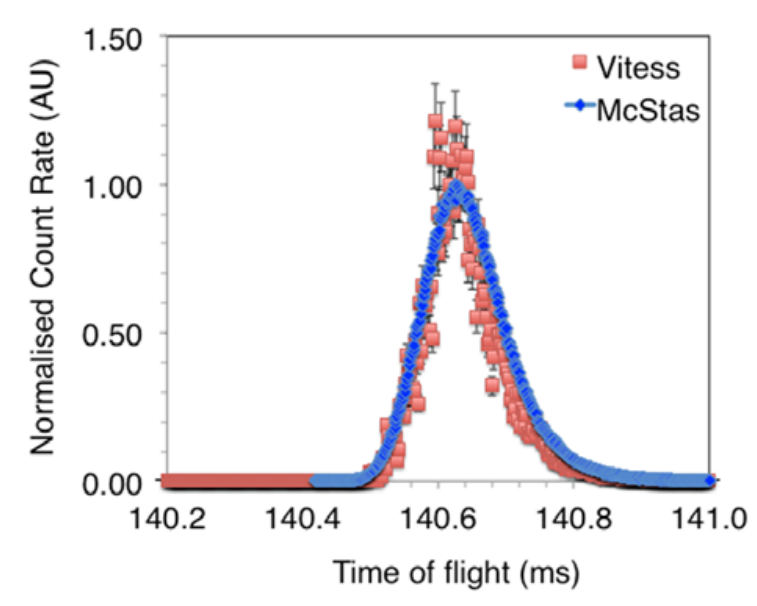

Figure 6. Time of flight data recorded by the detectors of the virtual BASIS instrument using VITESS and McStas.

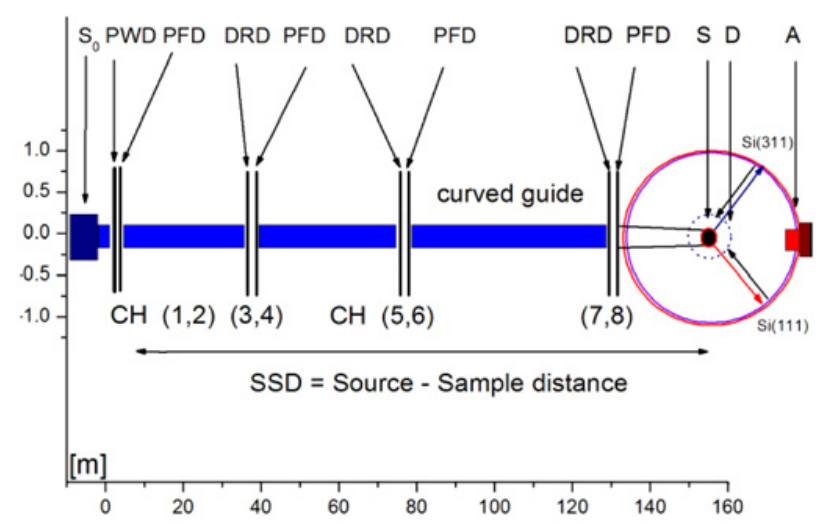

Figure 7. Schematic of the MIRACLES instrument to be proposed.

at the detector position. A comparison of experimental data (symbols) and simulation (solid lines) reveals that the Gaussian analyser describes well the energy response of the analyser both in terms of time distribution and peak position.

The BASIS instrument simulation results from VITESS as compared with McStas can be seen in Fig. 6. These were obtained from $10^{10}$ neutron trajectories and show good qualitative agreement with simulated McStas and the experimental BASIS data as shown in Fig. 5.

\section{Perspectives for MIRACLES}

After performing the simulations of the BASIS backscattering spectrometer, we have examined the elastic flux (neutrons that are scattered elastically) that can be expected when placing MIRACLES at the ESS.

The sample position is located at a distance of $156 \mathrm{~m}$ from the moderator surface (Fig. 7).

The flexibility of MIRACLES arises from the fact that a pulse width definition chopper (Fig. 7, PWD), placed close to the moderator, can be used to select any required portion from the long ESS pulse.

In this way, the total instrumental resolution can be approximated by the quadratic addition of the wavelength contribution of the analyser, see Eq. (6), the uncertainty of

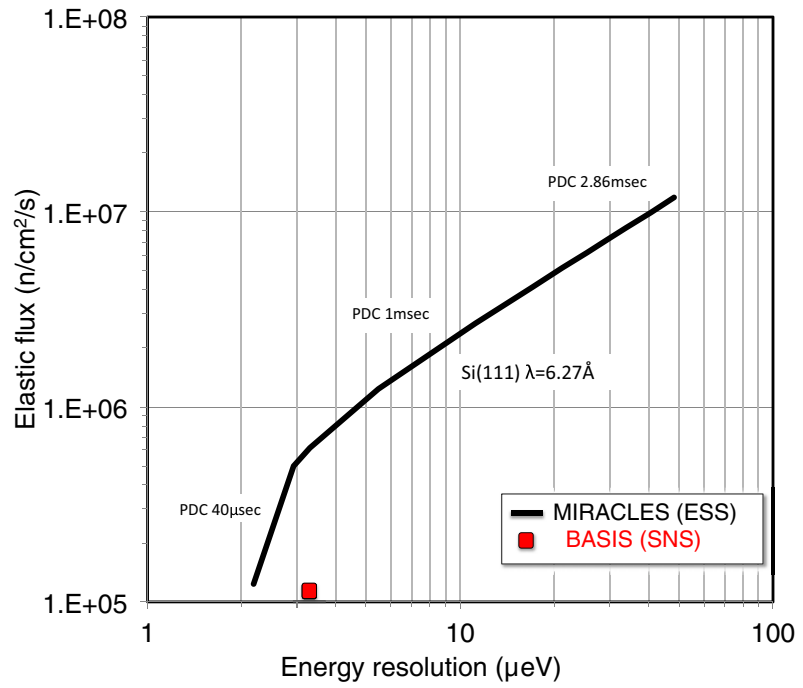

Figure 8. Flux versus elastic energy resolution for MIRACLES (solid black line) compared to BASIS (red square) [14]. The instrument energy resolution of MIRACLES can be varied, in the range of 3 to $32 \mu \mathrm{eV}$, by increasing the opening of the pulse width definition chopper (PDC). The $\mathrm{Si}(111)$ reflection is utilised by the analysers, which provides a wavelength of $6.267 \AA$ for a scattering angle of $2 \theta=88^{\circ}$.

the lattice spread, in Eq. (9), and the temporal uncertainty caused by the chopper pulse width $\tau$ in relation to the chopper period $T$.

$$
\left(\frac{\delta \lambda_{\text {instr }}}{\lambda}\right)^{2}=[\cot \theta \delta(\theta)]^{2}+\left[\frac{\delta(d)}{d}\right]^{2}+\left[\frac{\tau}{T}\right]^{2}
$$

Moreover, as shown by others [20], the spectral source peak brightness $B$ can be used to calculate the flux $\Phi$ at the sample position as shown in Eq. (17).

$$
\Phi=\frac{\mathrm{B} \times \Delta \Omega \times \Delta \lambda \times \Delta \mathrm{t}}{\tau} .
$$

Where, $\mathrm{B}$ is the ESS cold moderator brightness $2.7 \times$ $10^{13} \mathrm{n} /\left(\mathrm{cm}^{2} \times \mathrm{s} \times \mathrm{sr} \times \AA\right)$ centred at $\lambda=6.27 \AA[2] . \Delta \Omega$ is the source solid angle transported to the sample equals $4.05 \times 10^{-3}(\mathrm{sr}), \Delta \lambda$ is the wavelength range corresponding to $0.0022 \AA$ for analysers with $\frac{\delta(d)}{d}=3.5 \times$ $10^{-4} . \Delta \mathrm{t} / \mathrm{T}$ is the duty cycle, which is defined as the ratio of the opening time of the pulse-shaping chopper to the repetition period of the ESS source.

The analytical results for MIRACLES, also shown in Fig. 8, suggest that for an elastic resolution of $c a$. $3.5 \mu \mathrm{eV}$, approximately $6.3 \times 10^{5} \mathrm{n} /\left(\mathrm{cm}^{2} \mathrm{~s}\right)$ neutrons intersect the sample position. The solid black line illustrates the change of the elastic flux as a function of energy resolution; the energy resolution itself being adjusted by increasing the opening time of the window on the pulse definition chopper.

For the BASIS instrument, scaling the reported flux [14] to the current operating power of $1 \mathrm{MW}$ and integrating over the elastic peak results in an elastic flux for BASIS of ca $1.4 \times 10^{5} \mathrm{n} /\left(\mathrm{cm}^{2} \mathrm{~s}\right)$ (Fig. 8, red square). Therefore, a gain factor of 5 can be expected when compared to BASIS. This flux gain assumes conventional 
linearly tapered and straight optics similar to the BASIS instrument.

Subsequent simulations with elliptical focusing guides showed that a factor of 10 is to be expected for an energy resolution of $c a .3 .5 \mu \mathrm{eV}$.

Moreover, the analysis above assumes that only a single pulse can be extracted from each ESS pulse. With the use of specially designed chopper windows, multiple pulse trains from the ESS pulse can be extracted and further flux gains can be obtained in correspondingly smaller dynamic ranges.

Critically, the gain factors presented in this paper are based on the ESS moderator brilliance published in 2013 and presented in the TDR report [2].

As new moderator technologies, such as reduced height moderators or "pancake moderators" [21], come to fruition, even larger flux gains are foreseen.

In conclusion, we have presented new results for a virtual instrument description of the BASIS instrument, which could be achieved only after developing a new spherically bent crystal analyser component in McStas. In turn, others could use the component for benchmarking and teaching purposes of other backscattering spectrometers. The envisaged performance of the MIRACLES instrument, based on analytical calculations, was also briefly presented. These findings are fundamental to the conceptual design of MIRACLES, a unique high-resolution neutron spectrometer, which will open new scientific horizons for the elucidation of a plethora of phenomena seen in modern materials.

The authors would like to thank Ken H. Andersen and Arno Hiess at the European Spallation Source (ESS) for the useful discussions. We would like to thank Bernhard Frick for the long discussions on next generation backscattering during the IN16B commissioning. We are grateful to Ken Herwig, Eugene Mamontov and Niina Jalarvo for the long discussions about BASIS and it's science base at SNS. N.T. would especially like to thank Carolin Zendler (ESS) and Klaus Lieutenant for their assistance during the VITESS troubleshooting. We greatly appreciate the provision of the DMSC computer cluster at the Niels Bohr Institut, The University of Copenhagen for providing us the necessary resources for our simulations. Finally, we would like to extend our gratitude to Garrett E. Granroth at the SNS for his work on the SNS moderator for McStas.

\section{References}

[1] D. N. Argyriou and H. N. Bordallo, Nat. Mater. 8, 440 (2009)

[2] Technical Design Report for the European Spallation Source http://esss.se/documents/tdr/chapters/ TDR_Part3.pdf (2013), p. 92
[3] G. Schuck, R. E. Lechner, and K. Langer, Appl. Phys. A - Mater. Sci. Process. 74, S1098 (2002)

[4] A. Desmedt, R. E. Lechner, J.-C. Lassegues, F. Guillaume, D. Cavagnat, and J. Grondin, Solid State Ionics 252, SI, 19 (2013)

[5] R. E. Lechner, J. Fitter, N. A. Dencher, and T. Hauss, Physica B - Condens. Matter 385-386, 835 (2006)

[6] G. Ehlers, H. Casalta, R. E. Lechner, and H. Maletta, Neutron Spin Echo Spectrosc. Basics, Trends Appl. 601, 222 (2003)

[7] S. V Ghugare, E. Chiessi, B. Cerroni, M. T. F. Telling, V. G. Sakai, and G. Paradossi, Soft Matter 8, 2494 (2012)

[8] A. Deriu, M. T. Di Bari, and Y. Gerelli, Zeitschrift Fur Phys. Chemie-International J. Res. Phys. Chem. Chem. Phys. 224, 227 (2010)

[9] N. Tsapatsaris, S. Landsgesell, M. M. Koza, B. Frick, E. V Boldyreva, and H. N. Bordallo, Chem. Phys. 427, 124 (2013)

[10] N. Tsapatsaris, B. A. Kolesov, J. Fischer, E. V Boldyreva, L. Daemen, J. Eckert, and H. N. Bordallo, Mol. Pharm. 11, 1032 (2014)

[11] H. N. Bordallo, B. A. Zakharov, E. V. Boldyreva, M. R. Johnson, M. M. Koza, T. Seydel, and J. Fischer, Mol. Pharm. 9, 2434 (2012)

[12] W. P. Gates, H. N. Bordallo, L. P. Aldridge, T. Seydel, H. Jacobsen, V. Marry, and G. J. Churchman, J. Phys. Chem. C 116, 5558 (2012)

[13] J. Fischer, N. Tsapatsaris, E. de Paula, and H. N. Bordallo, Eur. Phys. J. Spec. Top. 223, 1831 (2014)

[14] E. Mamontov and K. W. Herwig, Rev. Sci. Instrum. 82, 10 (2011)

[15] B. Frick, E. Mamontov, L. van Eijck, and T. Seydel, Zeitschrift Fur Phys. Chemie-International J. Res. Phys. Chem. Chem. Phys. 224, 33 (2010)

[16] P. Willendrup, E. Farhi, and K. Lefmann, Phys. BCondensed Matter 350, E735 (2004)

[17] D. Wechsler, G. Zsigmond, F. Streffer, J. A. Stride, and F. Mezei, Phys. B 276, 71 (2000)

[18] Super-Mirror Reflectivity Data from Mirrotron http://www.mirrotron.kfkipark.hu/ supermirror.html (2014)

[19] J. Kulda, Acta Crystallogr. Sect. A 40, 120 (1984)

[20] F. Demmel and K. H. Andersen, Meas. Sci. Technol. 19, (2008)

[21] K. Batkov, A. Takibayev, L. Zanini, and F. Mezei, Nucl. Instruments Methods Phys. Res. Sect. aAccelerators Spectrometers Detect. Assoc. Equip. 729, 500 (2013) 